\title{
Raman study on defective graphene: Effect of the excitation energy, type, and amount of defects
}

\author{
Axel Eckmann, ${ }^{1}$ Alexandre Felten, ${ }^{2}$ Ivan Verzhbitskiy, ${ }^{2}$ Rebecca Davey, ${ }^{2}$ and Cinzia Casiraghi ${ }^{1,2, *}$ \\ ${ }^{1}$ School of Chemistry, University of Manchester, United Kingdom \\ ${ }^{2}$ Physics Department, Free University Berlin, D-14195 Berlin, Germany \\ (Received 11 February 2013; revised manuscript received 29 March 2013; published 15 July 2013)
}

\begin{abstract}
We present a detailed Raman study of defective graphene samples containing specific types of defects. In particular, we compared $s p^{3}$ sites, vacancies, and substitutional Boron atoms. We find that the ratio between the $D$ and $G$ peak intensities, $I(D) / I(G)$, does not depend on the geometry of the defect (within the Raman spectrometer resolution). In contrast, in the limit of low defect concentration, the ratio between the $D^{\prime}$ and $G$ peak intensities is higher for vacancies than $s p^{3}$ sites. By using the local activation model, we attribute this difference to the term $C_{S, x}$, representing the Raman cross section of $I(x) / I(G)$ associated with the distortion of the crystal lattice after defect introduction per unit of damaged area, where $x=D$ or $D^{\prime}$. We observed that $C_{S, D}=0$ for all the defects analyzed, while $C_{S, D^{\prime}}$ of vacancies is 2.5 times larger than $C_{S, D^{\prime}}$ of $s p^{3}$ sites. This makes $I(D) / I\left(D^{\prime}\right)$ strongly sensitive to the nature of the defect. We also show that the exact dependence of $I(D) / I\left(D^{\prime}\right)$ on the excitation energy may be affected by the nature of the defect. These results can be used to obtain further insights into the Raman scattering process (in particular for the $D^{\prime}$ peak) in order to improve our understanding and modeling of defects in graphene.
\end{abstract}

DOI: 10.1103/PhysRevB.88.035426

PACS number(s): 81.05.ue, 78.30.Ly, 78.67.Wj, 63.22.Rc

\section{INTRODUCTION}

Graphene has attracted enormous interest because of its unique properties. ${ }^{1-4}$ Near-ballistic transport at room temperature and high mobility ${ }^{1,5-8}$ make it a potential material for nanoelectronics. ${ }^{9-13}$ Furthermore, its optical and mechanical properties, combined with its high charge mobility, allow the use of this material for other applications, such as thinfilm transistors, transparent and conductive composites and electrodes, and opto-electronics. ${ }^{14-21}$

Graphene is usually considered as a perfect honeycomb crystal. However, real samples may contain defects. The amount and nature of defects strongly depend on the production method and may change from sample to sample. ${ }^{16,22-30}$ Defects can also be introduced in pristine graphene through ion bombardment, ${ }^{31-36}$ e-beam irradiation, ${ }^{37,38}$ soft $\mathrm{x}$-ray irradiation, ${ }^{39}$ covalent modification, ${ }^{40-53}$ and implantation of substitutional atoms. ${ }^{54-57}$ The possibility of introducing only well-defined defects and carefully control their amount allows fine tuning of the properties of graphene: defect lines can be used as metallic wire interconnectors for nanoelectronics, ${ }^{58,59}$ while $s p^{3}$-site defects can be used to turn the electronic properties of graphene from metallic to insulating, leading to the creation of stoichiometric graphene-based derivatives..$^{40,41}$ These can be easily obtained by exposing the crystal to a plasma. This method is very attractive for industrial applications because it is a simple, fast and scalable process. Depending on the plasma gas used, various species such as oxygen, fluorine, nitrogen, and chlorine can be grafted at the graphene scaffold. The bonding with the out-of-plane atom changes the carbon hybridization from $s p^{2}$ to $s p^{3}$, leading to changes in the electronic and optical properties. ${ }^{40,41}$

Raman spectroscopy is a fast and nondestructive technique for investigating the properties of graphene. ${ }^{60-63}$ This is able to identify graphene from graphite and few-layer graphene, ${ }^{64}$ and to probe doping level, ${ }^{65-67}$ strain, ${ }^{68,69}$ disorder, ${ }^{31-33,70,71}$ chemical derivatives, ${ }^{40-42}$ and the atomic arrangement at the edges. $^{72,73}$
Due to its sensitivity to defects, Raman spectroscopy has been used since more than 40 years to study disorder in carbon-based materials, from nanocrystalline graphite, ${ }^{74-82}$ to disordered carbons, ${ }^{83-85}$ and to carbon nanotubes. ${ }^{86-88}$ These works provided important advances in understanding disorder in $s p^{2}$-bonded carbon materials and they strongly contributed to the widespread use of Raman spectroscopy for general characterization of these samples. However, the correlation between defect-activated Raman features and geometry of defects is still missing. We do not know if Raman spectroscopy is sensitive to every type of defect, i.e., if and how the disorder-activated Raman intensities depend on the nature of the defects. For instance, Raman spectroscopy could be more sensitive to certain defects rather than others. If so, then a Raman spectrum without a $D$ peak would not be necessarily associated with a defect-free material.

To answer these questions, one needs to be able to carefully introduce defects in the material and control their nature, in order to compare the corresponding Raman spectra. In the case of a three-dimensional (3-D) material such as graphite, this is extremely challenging. The only defect that can be carefully controlled is the grain boundary, which depends on the size of the crystalline grains. Other 3-D materials such as disordered carbons do not appear suitable for this type of study because they contain both topological and structural disorder, that is disorder is not defined only by the $s p^{3}$ content, but also by the different amount and type of $s p^{2}$ clustering. ${ }^{83-85}$ In general, structural and topological disorders are not correlated with each other (e.g., two disordered carbons films may have the same $s p^{3}$ content, but a different degree of $s p^{2}$ clustering) and they strongly depend on the deposition conditions. ${ }^{83-85}$ This makes the Raman spectrum of disordered carbon rather difficult to analyze and correlate with the specific nature of disorder. Moving to low-dimensional carbon forms, nanotubes are difficult to manipulate and characterize, due to their one-dimensional nature. In contrast, graphene is an ideal material to study the Raman sensitivity to defects because its 
two-dimensional nature makes it easy to add, remove, or move carbon atoms, i.e., to carefully introduce only a specific type of disorder.

Here we present a detailed analysis of the evolution of the Raman spectra of graphene samples containing specific defects, such as $s p^{3}$ sites, substitutional atoms, and vacancies. We show that the general trend of the Raman spectrum of defective graphene does not depend on the nature of defects: a two-stage disordering evolution is always observed, no matter the geometry of the defect. In each stage the Raman fit parameters have a different dependence on the excitation energy. By comparing the Raman spectra of graphene containing $s p^{3}$ sites, substitutional atoms and vacancies, we found that in the limit of small defect concentration the $D$ peak is not sensitive to the defect geometry, but only to the amount of disorder (at least within the Raman resolution and for the type of defects studied here). In contrast, the $D^{\prime}$ peak shows a strong dependence on the type of defect introduced in the lattice, e.g., the $D^{\prime}$ peak intensity is higher for vacancies than $s p^{3}$ sites. Within the local activation model, ${ }^{33}$ we attribute this to the term $C_{S}$, representing the Raman cross section of $I(x) / I(G)$ associated with the distortion of the crystal lattice after defect introduction per unit of damaged area, where $x=D$ or $D^{\prime}$. Thus, this parameter is expected to strongly depend on the nature of the defect. ${ }^{33}$ We found that for the $D$ peak $C_{S} \sim 0$, no matter the nature of the defect; while for the $D^{\prime}$ peak, $C_{S}=0.33$ for $s p^{3}$ sites, and $C_{S}=0.82$ for vacancies. This makes the $D^{\prime}$ peak more sensitive to vacancies than $s p^{3}$ sites.

This paper is organized as follows: Sec. II presents the Raman scattering background, while Sec. III describes the experiential setup and the sample preparation. Section IV shows the evolution of the Raman spectrum with the amount of defects, its dependence on the excitation energy and on the nature of defects.

\section{BACKGROUND}

The Raman spectrum of graphene is composed of two main features, the $G$ and the 2D peaks, which lay at around 1580 and $2680 \mathrm{~cm}^{-1}$, respectively, when taken at an excitation energy of $2.4 \mathrm{eV}(514 \mathrm{~nm}){ }^{64}$ The $G$ peak corresponds to the $E_{2 g}$ phonon at the Brillouin zone center $\left(\boldsymbol{\Gamma}\right.$ point). ${ }^{74}$ The $2 \mathrm{D}$ peak is an overtone peak, associated with the breathing modes of six-atom rings. ${ }^{64}$ It comes from TO phonons in the vicinity of the $\boldsymbol{K}$ point $^{74}$ and it is activated by a resonant intervalley scattering process. ${ }^{89,90}$

Raman spectroscopy is able to probe defects in graphitic materials because, in addition to the $G$ and 2D peaks that always satisfy the Raman selection rule, ${ }^{91}$ the otherwise forbidden $D$ and $D^{\prime}$ bands appear. ${ }^{74,78}$ They correspond to single phonon intervalley and intravalley scattering events, respectively, where the defect provides the missing momentum in order to satisfy momentum conservation during the Raman scattering process. ${ }^{89,90,92}$ Another (weak) defect-activated peak is observed at about $3000 \mathrm{~cm}^{-1}$, corresponding to the combination mode of the $D$ and $D^{\prime}$ modes. It is therefore called the $D+D^{\prime}$ peak.

The activation mechanism of the defect-activated features, their overtones, and combination modes involves resonant electronic transitions. ${ }^{89,90,92}$ Hence the frequency of these peaks are intimately correlated to the electronic band structure making the peaks dispersive with the excitation energy. ${ }^{80,89,93,94}$

Previous works on defective graphene introduced the local activation model in order to explain the evolution of the Raman spectrum for increasing amount of defects. ${ }^{31-33}$ Within this model the intensity of any defect activated peak $I(x)$, where $x=D$ or $D^{\prime}$, as compared to the $G$ peak intensity $I(G)$, is given by ${ }^{33}$

$$
\begin{aligned}
\frac{I(x)}{I(G)}= & C_{A} \frac{\left(r_{A}^{2}-r_{S}^{2}\right)}{\left(r_{A}^{2}-2 r_{S}^{2}\right)}\left[e^{-\pi r_{S}^{2} / L_{D}^{2}}-e^{-\pi\left(r_{A}^{2}-r_{S}^{2}\right) / L_{D}^{2}}\right] \\
& +C_{S}\left[1-e^{-\pi r_{S}^{2} / L_{D}^{2}}\right] .
\end{aligned}
$$

This equation shows that the intensity of the defectactivated peak depends on two length scales, $r_{S}$ and $r_{A}$, which are the radii of two circular areas measured from the defect site. The first length, $r_{S}$, is the radius of the structurally disordered area around the defect, so it is expected to change from defect to defect. ${ }^{33}$ For distances larger than $r_{S}$ but shorter than $r_{A}$, the lattice structure is preserved, but the proximity to a defect causes a mixing of Bloch states near the $\boldsymbol{K}$ and $\boldsymbol{K}^{\prime}$ valleys of the graphene Brillouin zone, thus causing a breaking of selection rules, and leading to an enhancement of the $D$ band. ${ }^{31,33} r_{A}$ defines the disk where the $D$ peak scattering takes place and it defines the activated area. ${ }^{33}$ From a microscopic point of view, an electron/hole excitation will only be able to "see" the structural defect if it is created sufficiently close to it and if the excited electron (or hole) lives long enough for the defective region to be probed by Raman spectroscopy. ${ }^{31,33}$ Therefore, the distance $r_{A}-r_{S}=l_{x}$ represents the length traveled over the lifetime of the electron-hole pair, roughly given by $v_{F} / \omega_{x}$, where $v_{F}$ is the graphene Fermi velocity and $\omega_{x}$ is the peak frequency of either the $D$ or $D^{\prime}$ peak. ${ }^{31,33}$

$C_{A}$ depends only on the Raman mode, being roughly given by the ratio of the electron-phonon coupling between the two phonons considered. ${ }^{31,33} C_{S}$ is a factor assumed to depend only on the geometry of the defect for a fixed phonon mode.

Note that Eq. (1) can also be used for the intensity measured as integrated area. In any case, in the limit of low defect concentration, the use of intensity or integrated area is equivalent. ${ }^{31,33,71}$ Only in the high disorder regime it is more informative to decouple the peak intensity from the full width at half maximum. ${ }^{32}$

There are also physical models based on first principles and quantum mechanics that calculate the intensities of the Raman resonant features. In particular, a recent work $^{92}$ has been able to successfully reproduce numerous features of the Raman spectrum of graphene. We compare our experimental data with the results presented in Ref. 92, in particular the dependence of the Raman features on the excitation energy and amount of defects. However, the simple on-site and hopping perturbations used in Ref. 92 to simulate defects in graphene are not suitable to describe real defects. ${ }^{71}$ Because of that, we will use the activation model to explain our results of the dependence of the Raman intensities on the geometry of the defect. 


\section{A. Dependence on the amount of defects}

The only parameter that changes with defect concentration $\left(n_{D}\right)$ in Eq. (1) is $L_{D}$, being $n_{D}=10^{14} / \pi L_{D}^{2} \cdot{ }^{32}$ This equation gives a nonmonotonic evolution of $I(x) / I(G)$. This is the result of two competing mechanisms for the increase and decrease in the defect-activated bands. The increase in the activated area gives rise to an increase of the defect activated peak intensities; on the other side, an increase in the defect-activated area produces a decrease of the intensities. Therefore, one can identify two stages, where one mechanism dominates the other. ${ }^{31,33}$ The transition from Stage 1 to Stage 2 is typically observed when the mean distance between two defects $\left(L_{D}\right)$ is comparable to $l_{x} \cdot{ }^{31}$ Note that the stage terminology was first introduced for disordered carbons. ${ }^{83-85}$

Within the two-stage model, $C_{S}$ is the value of $I(x) / I(G)$ measured in the highly disordered limit. ${ }^{33}$ This is difficult to measure since both the $D$ and $D^{\prime}$ peak intensities decreases in Stage 2 and the $D^{\prime}$ peak, being close to the $G$ peak, merges with this peak.

If we now focus on Stage 1 (i.e., low defect concentration), the model shows that both the $D$ and $D^{\prime}$ peak intensities increase with increasing amount of defects. In particular, in the limit of low defect concentration, a Taylor expansion of Eq. (1) to the first order gives:

$$
\frac{I(x)}{I(G)}=C_{A} \frac{\pi\left(r_{A}^{2}-r_{S}^{2}\right)}{L_{D}^{2}}+C_{S} \frac{\pi r_{S}^{2}}{L_{D}^{2}} .
$$

The defect concentration for this stage is then given by

$$
n_{D}\left(\mathrm{~cm}^{-2}\right)=\frac{10^{14}}{\pi^{2}\left[C_{A}\left(r_{A}^{2}-r_{S}^{2}\right)+C_{S} r_{S}^{2}\right]} \frac{I(x)}{I(G)} .
$$

\section{B. Dependence on the geometry of the defect}

By looking at Eq. (2), the dependence of the peak intensities on the nature of the defects is given by $r_{S}$ and $C_{S}$, being $r_{A}=r_{S}+l_{x}$, where $l_{x}$ is fixed by the phonon mode and the excitation energy.

The only experimental works reporting data for those parameters are based on vacancies. ${ }^{31-33}$ In these works, the $D$ peak was extensively studied. The following parameters were reported for intensity measured as height: $r_{A} \sim 3 \mathrm{~nm}$, $r_{s} \sim 1 \mathrm{~nm}, C_{A}=4.2 . C_{S}$ has been reported being 0 or 0.87 in Refs. 32 and 33, respectively. Reference 32 claims that $C_{S}=0$ for the ideal case where the defect is the breakdown of the C-C bonds. However, $C_{S}$ may be different for other types of defects such as $s p^{3}$ sites, which do not break the network, but just produce a different arrangement of the carbon atoms. In any case, one should observe that the term $C_{S}$ has a minor influence in Stage 1, in particular in the limit of very low defect concentration. A change in $C_{S}$ from 0 to 0.87 produces variations well smaller than $10 \%$ on $I(D) / I(G)$ at the beginning of Stage 1. Therefore, if we focus on the low defect concentration regime, it is correct to assume $C_{S} \sim 0$, so Eq. (2) becomes:

$$
\frac{I(D)}{I(G)} \simeq \frac{\pi C_{A}\left(r_{A}^{2}-r_{S}^{2}\right)}{L_{D}^{2}}
$$

Therefore, the $D$ peak depends on the defect geometry only through $r_{S}$.

In this work, we analyze $s p^{3}$ sites, vacancies, and implanted atoms, so we do not expect $r_{S}$ to strongly change with the type of defect. Consequently, we expect the $D$ intensity not to be able to probe differences in the geometry of the defects because the Raman spectrometer is not enough sensitive (the typical error bar on a Raman intensity ratio is $10 \%-15 \%$ ). Thus, in the following we will use Eq. (1) to find the defect concentration from $I(D) / I(G)$.

In the case of the $D^{\prime}$ peak, its intensity follows qualitatively the same behavior as the $D$ peak, i.e., it increases in Stage 1 and decreases in Stage 2. However, the exact dependence on defect concentration is different. ${ }^{71}$ Indeed Ref. 31 shows that $C_{S}$ cannot be neglected for the $D^{\prime}$ peak, and that small variations on $C_{S}$ can produce strong changes in $I\left(D^{\prime}\right) / I(G)$, even at low defect concentrations.

In this work we aim at comparing the results obtained for vacancies with other type of defects, such as Boron substitutional atoms and $s p^{3}$ sites. In particular, we will investigate the intensity dependence of the $D$ and $D^{\prime}$ peak on the parameter $C_{S}$, which we will refer to as $C_{S, D}$ and $C_{S, D^{\prime}}$, respectively.

\section{EXPERIMENTAL METHOD}

We studied three types of defects in graphene:

(i) $s p^{3}$ defects. Pristine graphene samples were prepared by micromechanical exfoliation of single-crystal graphite flakes (Nacional de Graphite LTDA) on $\mathrm{Si} / \mathrm{SiO}_{x}$ substrates. The flakes were then placed in a purpose-built chamber, where they underwent an inductively coupled plasma at RF of $13.56 \mathrm{MHz}^{42}$ Defects were introduced by exposing pristine graphene to a mild $\mathrm{O}_{2}$ and $\mathrm{CF}_{4}$ plasma. ${ }^{42}$ The plasma treatments were performed at a power of $10 \mathrm{~W}$ and a pressure of 0.1 Torr. The amount of defects was tuned by changing the treatment time (between $5 \mathrm{~s}$ and $300 \mathrm{~s}$ ). More details of the process are described in Ref. 42. In addition, we fluorinated some exfoliated flakes by using the technique described in Ref. 41. In all cases, chemical modification was performed on pristine samples with no detectable $D$ peak.

(ii) Vacancylike defects. The samples were produced by anodic bonding, as reported in Refs. 24,71. We also used the results reported in Refs. 31-33, where graphene was exposed to $\mathrm{Ar}^{+}$ion bombardment.

(iii) Substitutional atoms. We used B-doped graphene samples, as reported in Ref. 54.

In the following discussion, we will group our data based on the nature of defects, so we will refer to "vacancies" as the data obtained by anodic bonding and ion bombardment, while we will refer to " $s p^{3}$ " as the data obtained for oxidized and fluorinated samples. The nature of the defects in these samples has been verified in Refs. 33,42,71.

Raman measurements were performed in a backscattering configuration with a confocal WITec spectrometer equipped with $2.54 \mathrm{eV}, 2.41 \mathrm{eV}$, and $1.96 \mathrm{eV}$ laser lines. The WITec spectrometer is also equipped with a piezoelectric stage that allows Raman mapping of areas up to $200 \times 200 \mu \mathrm{m}^{2}$. Because of the inhomogeneity of the fluorinated and anodic bonded flakes, we used Raman mapping to collect a large 
amount of spectra with varying $I(D) / I(G)$ ratios, typically between 0.5 and 4 . Multiwavelength analysis was performed with a Dilor triple-monochromator Raman spectrometer, equipped with an $\mathrm{Ar}-\mathrm{Kr}$ laser with excitation lines between 647 and $457 \mathrm{~nm}$. In all cases we used a 100× objective giving a laser spot size of about $400 \mathrm{~nm}$. The laser power was kept well below $1 \mathrm{~mW}$ to avoid damage or heating, which could induce desorption of the adatoms from graphene. The spectral resolution is $\simeq 2 \mathrm{~cm}^{-1}$. The $D, G$, and $2 D$ peaks were fitted with Lorentzian functions and the $D^{\prime}$ peak by a Fano line shape. A Fano line shape was preferred to a Lorentzian because for a defect concentration close to the transition between Stage 1 and Stage 2 and beyond this point, the $G$ and $D^{\prime}$ peak start to merge. This does not allow using a fully symmetric line for fitting the $G$ peak. In any case, the use of Fano or Lorentzian line does not change the results relying on the peaks' amplitude as the peak height is the same for the two lines. We analyze the following Raman fit parameters: position (POS) and full width at half maximum (FWHM), and intensities. Here, we refer to peak intensity as the height of the peaks and it will be denoted as $I(D), I(G), I\left(D^{\prime}\right), I(2 D)$ for the $D, G, D^{\prime}$, and $2 D$ peaks, respectively. The integrated areas will be labeled $\mathrm{A}(D)$, $\mathrm{A}(G), \mathrm{A}\left(D^{\prime}\right)$, and $\mathrm{A}(2 D)$.

Note that the $D^{\prime}$ peak has a small intensity compared to the $D$ peak; often the peak appears just as a small shoulder of the $G$ peak. However, at low and moderate defect concentration, the $D^{\prime}$ peak can be clearly distinguished from the $G$ peak and it can have relatively large intensity (up to $1 / 3$ of the intensity of the $G$ peak).

\section{RESULTS AND DISCUSSION}

\section{A. Evolution of the Raman spectrum with the amount of defects}

Let us start by looking at the evolution of the Raman spectrum of graphene for several types of defective graphene with increasing defect concentration.

Figures 1(a) and 1(b) show a collection of first and second order Raman spectra, measured at $2.41 \mathrm{eV}$ of graphene containing $s p^{3}$ sites obtained by partial fluorination (a) and oxidization (b). Figure 1(c) shows a collection of first and second order Raman spectra for increasing defect concentration (from bottom to top), measured at $2.41 \mathrm{eV}$ of graphene with vacancylike defects obtained by anodic bonding as described in Ref. 24.

The defect-activated features $\left(D, D^{\prime}\right.$, and $D+D^{\prime}$ peaks $)$ appear in all the spectra. Qualitatively, one can see that as the defect concentration increases, the $D$ peak increases at first and then decreases while broadening. The $D^{\prime}$ peak increases and eventually merges with the $G$ peak and the $2 D$ peak monotonously decreases until it almost disappears. The $D+D^{\prime}$ peak increases in intensity and broadens. The same general evolution is observed in ion-bombarded graphene. ${ }^{31-33}$

Figure 2 shows the evolution of the Raman fit parameters against exposure time for the oxidized samples. Although we do not know the exact relation between plasma exposure time and defect concentration, we expect the amount of defects to increase for increasing time.

If we focus on the $D$ peak intensity [Fig. 2(a), top], we can clearly see a two-stage evolution: at low defect concentration (between 0 and $40 \mathrm{~s}), I(D)$ and $I\left(D^{\prime}\right)$ increase for increasing
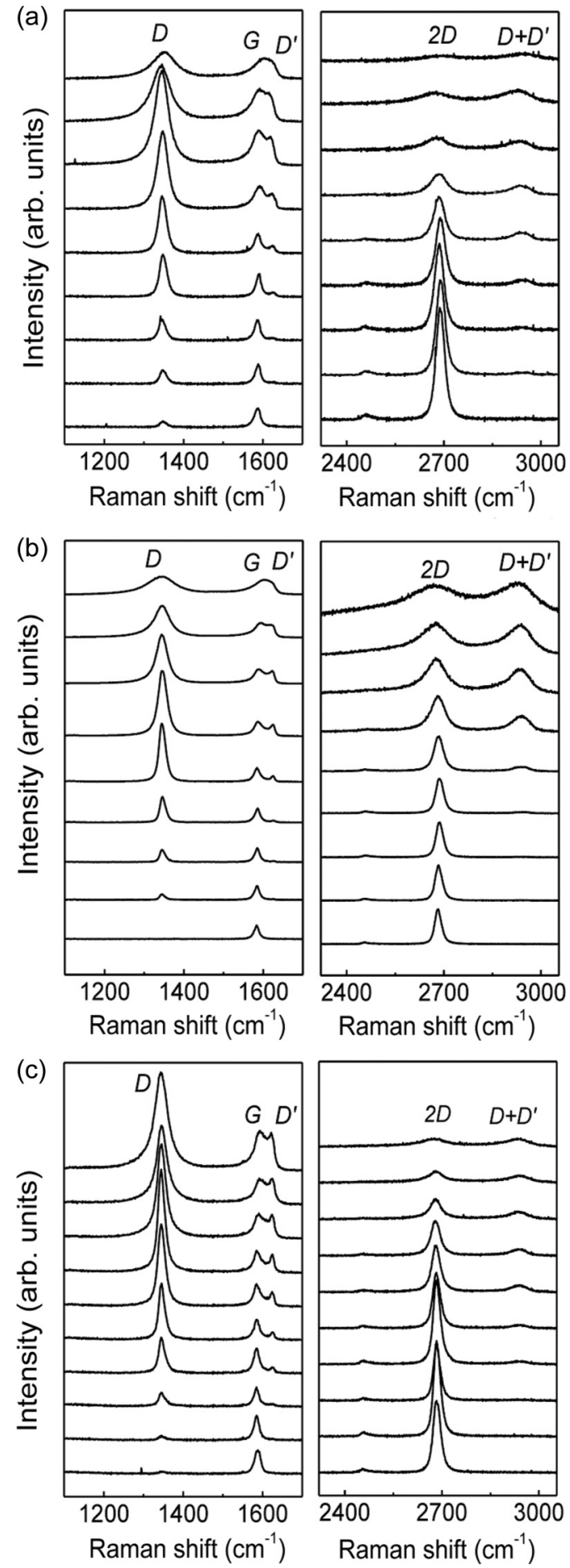

FIG. 1. Representative spectra of (a) fluorinated graphene, (b) oxidized graphene, and (c) anodic bonded graphene, with increasing defect concentrations (from bottom to top). All measurements are taken at $2.41 \mathrm{eV}$.

time. This corresponds to Stage 1. In contrast, $I(G)$ and $I(2 D)$ show very little variation. At higher defect concentration (between 40 and $300 \mathrm{~s}$ ), $I(D)$ decreases with time. This corresponds to Stage 2. Furthermore, $I(G)$ decreases, but more slowly than $I(D)$ and $I(2 D)$ strongly decreases. The transition between the two stages corresponds to the maximum $I(D) / I(G)$ 

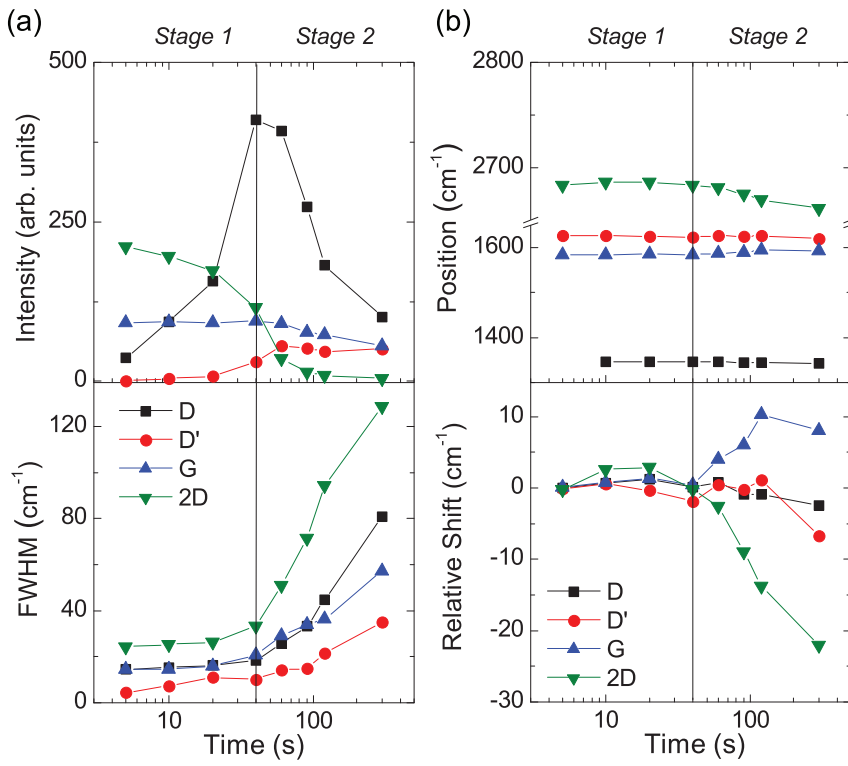

FIG. 2. (Color online) Evolution of (a) intensity and FWHM of the $D, D^{\prime}, G$, and $2 D$ peaks for increasing defect concentration. (b) Absolute position of the $D, D^{\prime}, G, 2 D$ peak and relative shift of these peaks, as compared with the position measured on pristine graphene. Measurements taken at $2.41 \mathrm{eV}$.

$(\simeq 5)$. This two-stage evolution agrees with the experimental results obtained in vacancy-defective graphene. ${ }^{31-33}$

A two-stage evolution is also visible when looking at the peaks' FWHM [Fig. 2(a), bottom]. In Stage 1, the FWHM of all peaks remains constant, while it strongly increases in Stage 2. The FWHM of any peak can consequently be used to distinguish between the two stages. Conclusions drawn about the defect concentration when only considering the ratio $I(D) / I(G)$ is indeed ambiguous since a given $D$ peak intensity [or $I(D) / I(G)$ ratio] may correspond to two different defects concentrations.

The introduction of disorder also changes the peak positions, as shown in Fig. 2(b) (top). In order to better visualize these changes, we plotted the shift of the position with respect to the positions measured on the pristine graphene [Fig. 2(b), bottom]. This figure shows that the $D, D^{\prime}$, and $2 D$ peak positions down-shift for increasing defect concentration, where $\operatorname{Pos}(2 D)$ experiences the largest shift (well above $15 \mathrm{~cm}^{-1}$ at $300 \mathrm{~s}$ ). In contrast, the $G$ peak shifts to higher wave numbers, up to $10 \mathrm{~cm}^{-1}$ at $300 \mathrm{~s}$. We expect the $G$ peak to shift with disorder: This effect is well reported for disordered carbons. ${ }^{83-85}$ Changes in the $G$ peak position occur because the introduction of defects relaxes the Raman selection rule $(\boldsymbol{q} \simeq 0)$. Due to the Kohn anomaly, ${ }^{95}$ the phonon energy strongly increases with the phonon wave vector, resulting in a blue-shift of the $G$ peak position for increasing disorder in the hexagonal rings. Note that at $300 \mathrm{~s}$, the $D^{\prime}$ and the $G$ peak have merged, so the uncertainty related to the fit is large. The down-shift of both the $D$ and $2 D$ peaks is assigned to the TO phonon dispersion branch in the vicinity of the $\boldsymbol{K}$ point while $\operatorname{Pos}\left(D^{\prime}\right)$ decreases due to the LO phonon branch dispersion near $\Gamma{ }^{31}$ These observations agree with the results from Refs. 31,47.

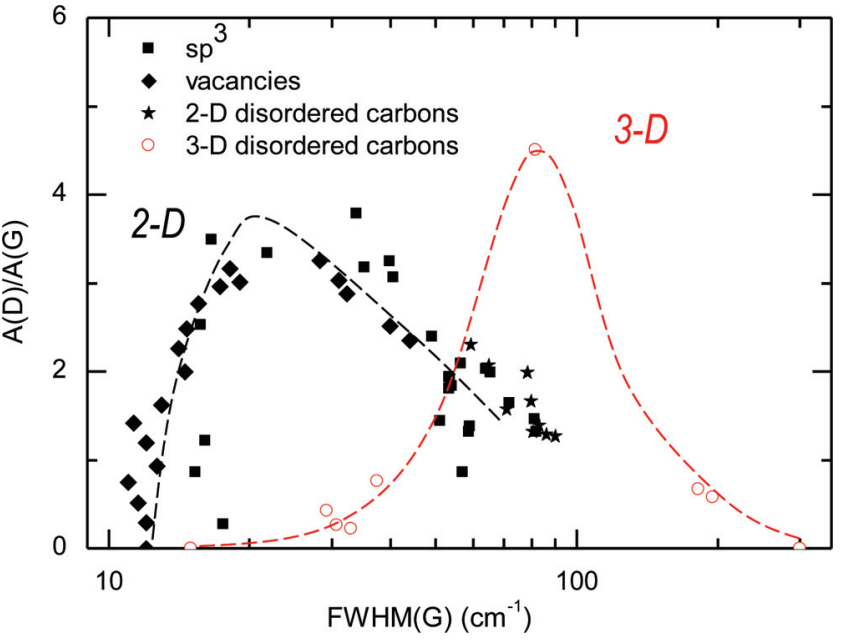

FIG. 3. (Color online) $A(D) / A(G)$ against $\operatorname{FWHM}(G)$ for a wide collection of two-dimensional (2-D) defective graphene, ${ }^{27,28,31,96,97}$ as compared with the three-stage evolution of disordered 3-D carbons. ${ }^{78,85}$ The dotted lines are guides to the eyes.

It is now interesting to compare the disordering trajectory of the Raman spectrum of disordered graphene and disordered carbons, i.e., to compare disorder in two- and three-dimensional carbon-based materials.

Figure 3 plots the ratio $A(D) / A(G)$ against $\operatorname{FWHM}(G)$. This allows one to decouple the amount of defective hexagonal rings from the overall disorder. ${ }^{85}$ Indeed, the FWHM always increases for increasing disorder because this parameter is sensitive to all types of defects, either in the $s p^{2}$ rings or chains; in contrast, $A(D) / A(G)$ is sensitive only to defects in the rings. Note that we decided to plot the ratio $A(D) / A(G)$ because it allows comparison of a large set of data available in the literature, which is reported in area only.

The two-dimensional materials group includes fluorinated graphene $\left(\right.$ defect $=s p^{3}$ site), ion-bombarded graphene (defect $=$ vacancies $),{ }^{31}$ and graphene oxide $(\mathrm{GO})$ and reduced graphene oxide (rGO). ${ }^{27,28,96,97}$ These last two materials have been selected because in contrast to hydrogenated and fluorinated graphene that contain only $s p^{3}$ sites, GO and rGO contain different types of defects, whose nature and corresponding amount is not completely known. From this point of view, GO and $\mathrm{rGO}$ can be seen as the two-dimensional equivalent of disordered carbons. For the three-dimensional materials group, we used the data reported for disordered carbons in Ref. 85.

Figure 3 shows that both two- and three-dimensional disordered materials have a similar "bell-like" disordering trajectory, in agreement with the two-stage evolution described in Sec. II. However, disordered carbon material extends into a third stage [for $\operatorname{FWHM}(G)>200 \mathrm{~cm}^{-1}$ ], which corresponds to the conversion of the rings into $s p^{2}$ chains. ${ }^{83-85}$ It does not seem to happen for graphene, even in highly fluorinated samples, so the defects may stretch the rings but do not open them into chains. In the following discussion, we will use Eq. (1) to calculate the defect concentration from $I(D) / I(G)$ for all defective two-dimensional samples based on the fact that they observe the same disordering trajectory, as seen in Fig. 3. 

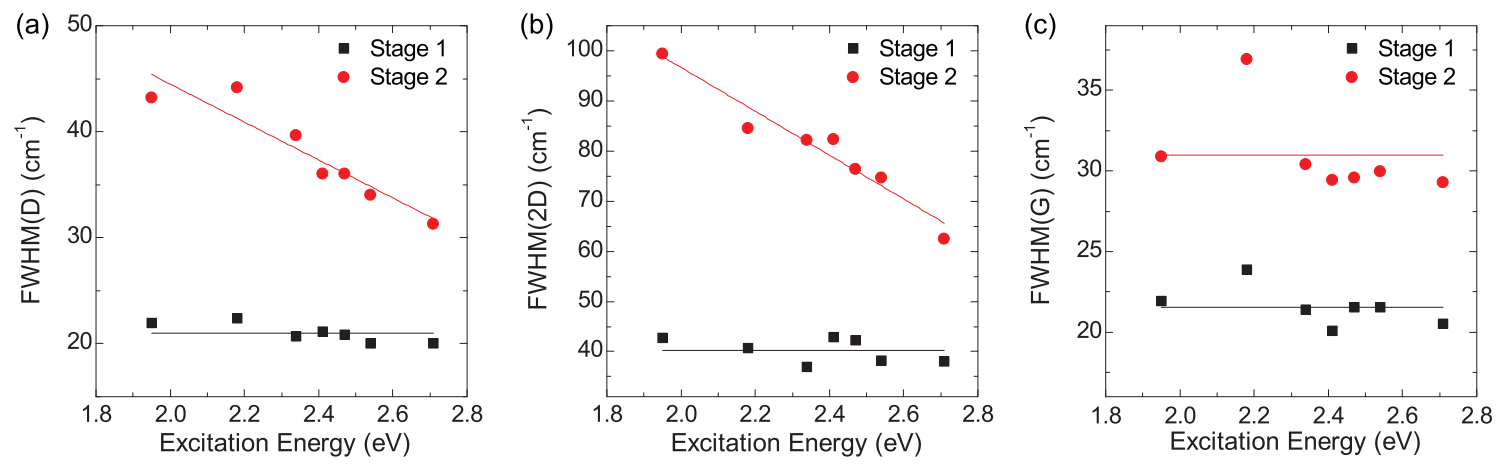

FIG. 4. (Color online) Evolution of the FWHM of the (a) $D$ peak, (b) $2 D$ peak, (c) $G$ peak versus excitation energy for Stage 1 and Stage 2 samples. The solid lines are fits of the experimental data.

In conclusion, we have observed a similar two-stage evolution of the Raman fit parameters for graphene containing vacancy and $s p^{3}$ sites. Neither the position nor the FWHM of the peaks is strongly sensitive to the type of defects.

\section{B. Multiwavelength Raman analysis}

The previous analysis was performed at a fixed wavelength of $2.4 \mathrm{eV}$. We now extend the analysis to other excitation lines in the visible range. We select two oxidized graphene samples with two different defect concentrations: One sample shows $I(D) / I(G)=4$ and $\operatorname{FWHM}(G)=20 \mathrm{~cm}^{-1}$ at $2.4 \mathrm{eV}$; it therefore belongs to the onset of the stage transition but still in Stage 1, while the other sample shows $I(D) / I(G)=2.5$ and $\operatorname{FWHM}(G)$ $=43 \mathrm{~cm}^{-1}$ at $2.4 \mathrm{eV}$. Thus, it is representative of Stage 2 . Figures 4, 5, and 6 compare the evolution of several Raman fit parameters of these two stage-reference samples.

Figure 4 shows that at Stage $1, \operatorname{FWHM}(D), \operatorname{FWHM}(2 D)$, and $\operatorname{FWHM}(G)$ do not significantly change with excitation energy. However, at Stage 2, the FWHM of the first and second order $D$ peak strongly decrease with increasing excitation energy at a rate of -18 and $-43 \mathrm{~cm}^{-1} / \mathrm{eV}$ for the $D$ and the $2 D$ peak, respectively. In contrast, $\operatorname{FWHM}(G)$ is constant in Stage 2. The FWHM of all the peaks in Stage 2 is larger than the FWHM in Stage 1, as already shown in Fig. 2(a). In comparison, Ref. 32 reported $-20 \mathrm{~cm}^{-1} / \mathrm{eV}$ and $-53 \mathrm{~cm}^{-1} / \mathrm{eV}$ for the $D$ and the $2 D$ peak, respectively, for a Stage 2 ion-bombarded sample. The same behavior has been observed also for the $D$ peak of disordered carbons ${ }^{83-85}$ and it has been attributed to the resonant mechanism in the presence of a broad distribution of defects: ${ }^{83-85}$ This is possible only in Stage 2, where the hexagonal crystal lattice becomes strongly deformed, i.e., can be seen as a disordered network containing small graphitic islands of different size. Thus, attention to the laser line must be paid when comparing the FWHM of the peaks measured in Stage 2.

Figure 5 shows the dispersion of the $D, 2 D$, and $D^{\prime}$ peaks. This is caused by the combination of the resonant scattering mechanism and the Kohn anomaly close to the $\boldsymbol{K}$ point. ${ }^{89,95}$ The slopes obtained for the $D$ peak are $48 \mathrm{~cm}^{-1} / \mathrm{eV}$ and $50 \mathrm{~cm}^{-1} / \mathrm{eV}$ for Stages 1 and 2, respectively. For the $2 D$ peak, we found a slightly different slope between the two stages: $101 \mathrm{~cm}^{-1} / \mathrm{eV}$ and $112 \mathrm{~cm}^{-1} / \mathrm{eV}$ for Stage 1 and Stage 2, respectively, which are similar to reported values obtained for nano-crystalline graphite. ${ }^{92,98}$ The red shift of both the $D$ and $2 D$ peaks in Stage 2 has been observed and attributed to changes in the phonon dispersion and the band structure of graphene ${ }^{47}$ until a band gap eventually opens up as expected for both hydrogenated or oxidized graphene. ${ }^{99,100}$

The $D^{\prime}$ peak also shows dispersion with the laser excitation, but its dependence is weaker compared to the $D$ peak, as previously observed in graphite. ${ }^{101}$ We do not show the position of the $D^{\prime}$ peak at Stage 2 because here the $G$ and the $D^{\prime}$ peaks merge and their positions are therefore not reliable.

Figure 6 compares the dependence of the ratio $I(D) / I(G)$ with excitation energy. $I(D) / I(G)$ strongly depends on the excitation energy for both stages. In Stage 1, since $I(G)$ is constant, the increase of $I(D) / I(G)$ with the excitation energy $E_{L}$ has to be attributed to $I(D)$ only. In the model used in Refs. 31-33 to
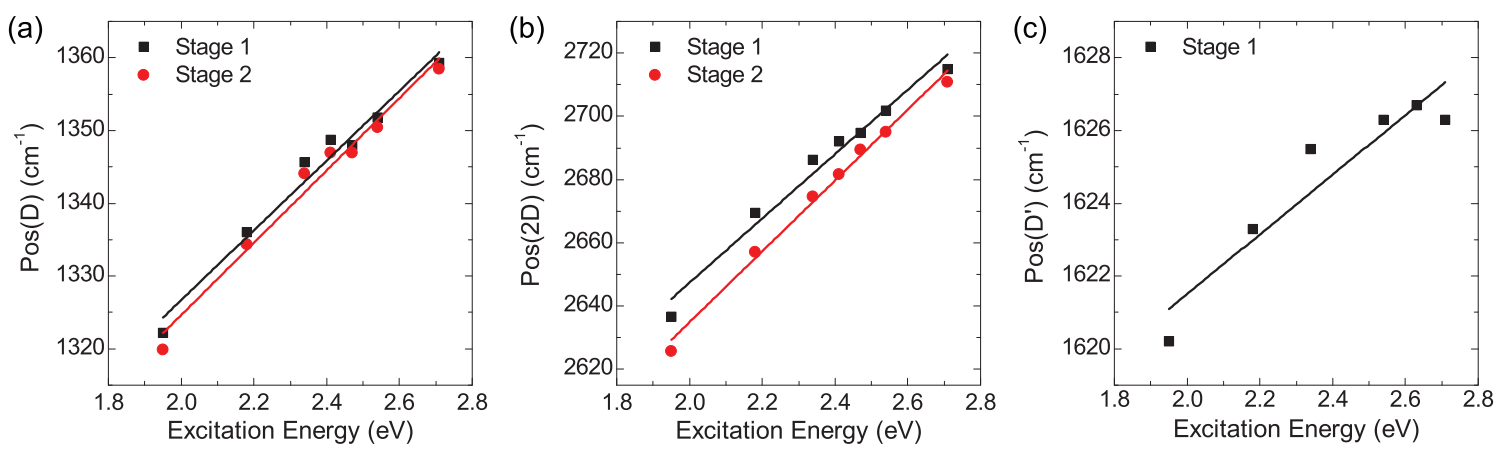

FIG. 5. (Color online) Evolution of the position of the (a) D peak, (b) 2D peak, (c) $\mathrm{D}^{\prime}$ peak versus excitation energy for Stage 1 and Stage 2 samples. The solid lines are linear fits of the experimental data. 


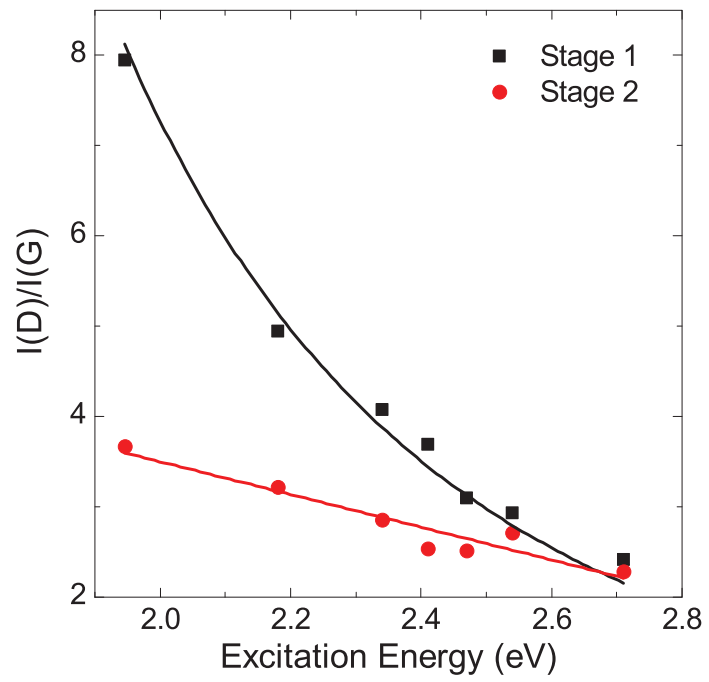

FIG. 6. (Color online) Evolution of the intensity ratio $I(D) / I(G)$ versus excitation energy for Stage 1 and Stage 2 samples. The black line is a fit following an $E_{L}^{-4}$ relation, while the red line is a linear fit.

describe defects obtained by ion bombardment, $I(D) / I(G)$ was found to be proportional to $E_{L}^{-4}$ for Stage 1 . The same trend is observed here with oxidized graphene and $I(D) / I(G)$ could be fitted well with the same equation. However, the evolution of $I(D) / I(G)$ for Stage 2 is different: This parameter depends linearly on the excitation energy. The breakdown of the $E_{L}^{-4}$ dependence at high defect concentration is due to the confinement of ordered $s p^{2}$ regions whose size becomes comparable to the average distance an electron hole travels before being scattered by a phonon. ${ }^{47}$ The same behavior was observed in graphitic foams in the same energy range. ${ }^{102}$ Our linear fit gives a slope of $-0.27 \mathrm{eV}^{-1}$, which is in excellent agreement with the result $\left(-0.31 \mathrm{eV}^{-1}\right)$ obtained in Ref. 102. The same behavior was observed for fluorinated graphene samples.

To summarize, we have shown that FWHM and relative intensities have a different behavior with the excitation energy depending on the disordering stage. Furthermore, the $E_{L}^{-4}$ dependence for $I(D) / I(G)$ in Stage 1 is the same both for graphene with vacancies or $s p^{3}$ sites.

\section{Dependence on the nature of defects}

We now compare the dependence on the defect concentration of the Raman intensities of two different types of defective graphene in Stage 1, one containing $s p^{3}$ sites, and the other with vacancies (Fig. 7). In this case, we also added the calculation from Ref. 92, which only reports the Raman intensities as an integrated area.

In the following discussion, we report the defect concentration as calculated from $I(D) / I(G)$ by using Eq. (1).

Figure 7 shows an overall good agreement between the calculations and the experimental data: $A(D)$ increases with defect concentration, while $A(2 D)$ decreases due to the increasing of the electron-defect scattering rate ${ }^{90,92}$ It is striking to observe that within the Raman resolution, the disordering trajectory for the $2 D$ and the $D$ peak areas for $s p^{3}$ sites and for vacancies overlap quite well, further suggesting that the Raman spectrometer is not able to probe small changes in $r_{S}$, at least

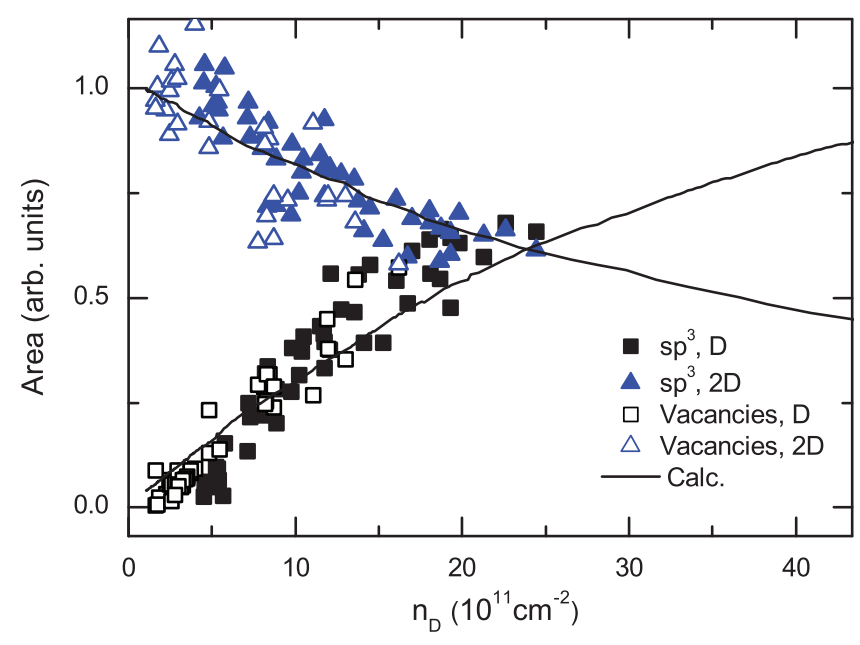

FIG. 7. (Color online) Evolution of the integrated area of the $2 D$ and the $D$ peaks as a function of the defect concentration for graphene flakes with either vacancies or $s p^{3}$ sites. The solid lines are taken from the calculations of Ref. 92.

for the type of defects here analyzed. Therefore, $I(D) / I(G)$ can be used to derive the amount of disorder [Eq. (1)], but it cannot provide any additional information on defects.

As highlighted in Sec. II, the data of Ref. 31 suggests for the $D^{\prime}$ peak a stronger dependence on the geometry of the defects, as compared to the $D$ peak, since $C_{S, D^{\prime}}$ cannot be neglected. Therefore, let us focus on the $D^{\prime}$ peak in the limit of low defect concentration, where Eq. (2) is valid.

Figure 8 compares $I\left(D^{\prime}\right) / I(G)$ as a function of $L_{D}$ for our samples, containing $s p^{3}$ sites, and the ones reported in Ref. 31, which contain vacancies. This figure shows that for the same defect concentration, $I\left(D^{\prime}\right) / I(G)$ is higher for vacancies than $s p^{3}$ sites. The solid lines are fits obtained using Eq. (1). In the fit we took $C_{A, D^{\prime}}$ equal to the ratio between the electron phonon coupling between the $D^{\prime}$ and the $G$ peaks optical phonons. ${ }^{33}$ By using phonon dispersion of Ref. 92 and scaling the electronic gap at the $M$ point to $4.6 \mathrm{eV}$ obtained by Ref. 103, we found that $C_{A, D^{\prime}} \sim 0.5$.

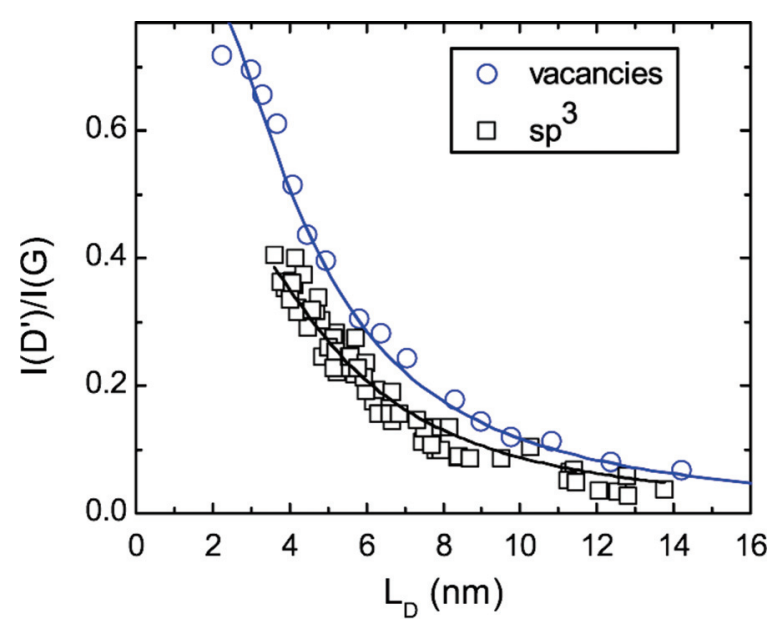

FIG. 8. (Color online) Evolution of the ratio $I\left(D^{\prime}\right) / I(G)$ for graphene flakes with either vacancies ${ }^{31}$ or $s p^{3}$ sites. The solid lines are fits obtained using Eq. (1). 


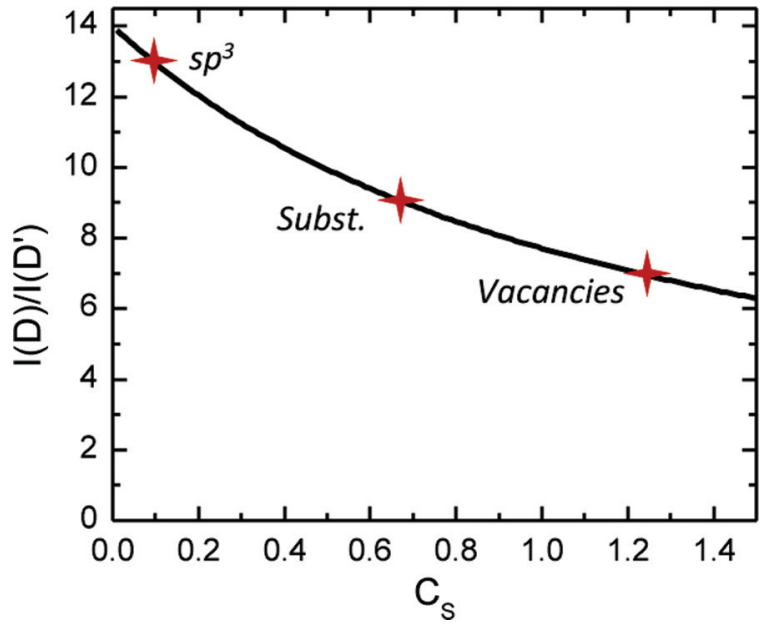

FIG. 9. (Color online) Plot of $I(D) / I\left(D^{\prime}\right)$ as a function of $C_{S, D^{\prime}}$ according to Eq. (7), valid in Stage 1.

The fit for graphene samples containing vacancies gives $C_{S, D^{\prime}}=0.82, r_{A, D^{\prime}}=2.6 \mathrm{~nm}$, and $r_{S, D^{\prime}}=1.4 \mathrm{~nm}$. Note that the values of $r_{A, D^{\prime}}$ and $r_{S, D^{\prime}}$ are in good agreement with the parameters found from the $D$ peak intensity (Sec. II).

If we now move to $s p^{3}$ by fitting the data with $r_{A, D^{\prime}}=2.6$ $\mathrm{nm}$ and $r_{S, D^{\prime}}=1.4$, we found $C_{S, D^{\prime}}=0.33$. Thus, $C_{S, D^{\prime}}$ for vacancies is almost 3 times larger than $C_{S, D^{\prime}}$ for $s p^{3}$ sites, i.e., the $D^{\prime}$ peak is more sensitive to vacancies than $s p^{3}$ sites.

In our previous work, ${ }^{71}$ we showed that the intensity ratio between the $D$ and $D^{\prime}$ peak can be used to identify the nature of defects at low defect concentration $\left(n_{D}<10^{12} \mathrm{~cm}^{-2}\right)$. In particular we found $I(D) / I\left(D^{\prime}\right) \simeq 7$ for vacancies and $\simeq 13$ for $s p^{3}$ sites (measured at $2.41 \mathrm{eV}$ ). We will show now that this dependence is strictly related to $C_{S, D^{\prime}}$. By using Eq. (2), we have:

$$
\begin{gathered}
\frac{I(D)}{I(G)} \simeq \frac{\pi\left[C_{A, D}\left(r_{A, D}^{2}-r_{S, D}^{2}\right)\right]}{L_{D}^{2}}, \\
\frac{I\left(D^{\prime}\right)}{I(G)} \simeq \frac{\pi\left[C_{A, D^{\prime}}\left(r_{A, D^{\prime}}^{2}-r_{S, D^{\prime}}^{2}\right)+C_{S, D^{\prime}} r_{S, D^{\prime}}^{2}\right]}{L_{D}^{2}} .
\end{gathered}
$$

By dividing Eq. (5) for Eq. (6), we get:

$$
\frac{I(D)}{I\left(D^{\prime}\right)} \simeq \frac{C_{A, D}\left(r_{A, D}^{2}-r_{S, D}^{2}\right)}{C_{A, D^{\prime}}\left(r_{A, D^{\prime}}^{2}-r_{S, D^{\prime}}^{2}\right)+C_{S, D^{\prime}} r_{S, D^{\prime}}^{2}} .
$$

This shows that $I(D) / I\left(D^{\prime}\right)$ strongly depends on $C_{S, D^{\prime}}$, making it sensitive to the geometry of the defect. Note that if $C_{S, D^{\prime}}=0$, then $I(D) / I\left(D^{\prime}\right)$ would be constant within the Raman resolution, no matter the geometry of the defects, in contrast to the results presented in Ref. 71 .

Figure 9 plots Eq. (7), obtained by using the values obtained by Ref. 33 for the $D$ peak $\left(r_{S, D}=1 \mathrm{~nm}, r_{A, D}=3 \mathrm{~nm}\right.$, $C_{A, D}=4.2$ ), and $r_{S, D^{\prime}}=1.4 \mathrm{~nm}, r_{A, D^{\prime}}=2.6 \mathrm{~nm}$ for the $D^{\prime}$ peak. This plot shows that the higher $C_{S, D^{\prime}}$, the larger the sensitivity of the $D^{\prime}$ peak intensity to a particular defect. From Fig. 9 we found that $C_{S, D^{\prime}} \simeq 0.1$ for $s p^{3}$ sites and $C_{S, D^{\prime}} \simeq 1.2$ for vacancies, in relatively good agreement with the values obtained by fitting the data in Fig. 8. Note that in the case of B-doped graphene, we found $I(D) / I\left(D^{\prime}\right) \simeq 9$, which gives $C_{S, D^{\prime}} \simeq 0.68$. In theory, one could see the $\mathrm{B}$ atom as a vacancy,

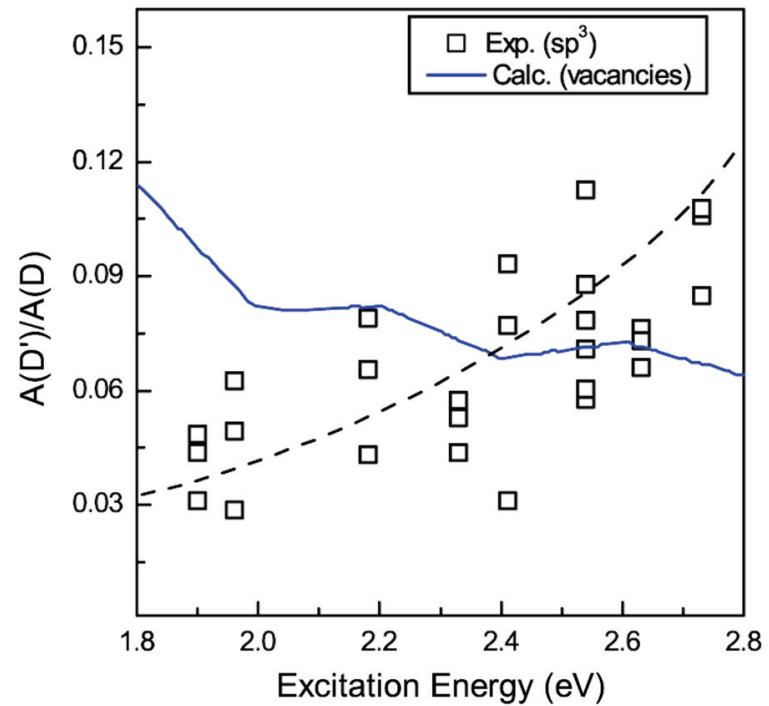

FIG. 10. (Color online) Ratio of the integrated areas under $D^{\prime}$ and $D$ peaks as a function of the excitation energy for graphene flakes with $s p^{3}$ sites (Stage 1). The solid line is the calculated evolution taken from Ref. 92 for vacancies defects. The dashed line is a guide to the eyes.

since this is equivalent to the lack of a carbon atom. On the other side, the introduction of B also deforms the crystal lattice being $\mathrm{B}$ bigger than $\mathrm{C}$. Furthermore, $\mathrm{B}$ is known to cluster for increasing B concentration, ${ }^{54}$ so this may explain why $I(D) / I\left(D^{\prime}\right)$ does not correspond with the value reported for vacancies.

Finally, it is important to investigate the dependence of the intensity of $I(D) / I\left(D^{\prime}\right)$ on the excitation energy: $I(D) / I(G)$ shows a well-known dependence on the laser energy. ${ }^{32}$ This is due to $C_{A}$, i.e., with the ratio between the electron-phonon coupling of the phonon at $\boldsymbol{K}(D$ peak) and at $\boldsymbol{\Gamma}$ ( $G$ peak). A similar argument is valid for the $D^{\prime}$ peak.

Figure 10 shows the ratio of the integrated areas for graphene flakes with $s p^{3}$ sites against the laser excitation energy. Here, we plot the integrated area ratio $A\left(D^{\prime}\right) / A(D)$ in order to compare our data with the calculations of Ref. 92, obtained for idealized vacancies defects. Note that in Stage 1, the FWHM of both the $D$ and the $D^{\prime}$ peaks did not change significantly. Thus, the use of area or intensity ratios is equivalent. This figure clearly shows that $A\left(D^{\prime}\right) / A(D)$ increases for increasing excitation energy for $s p^{3}$ sites, in contrast to vacancies. Therefore, the excitation energy dependence may be affected by the nature of the defects [see $C_{S, D^{\prime}}$ in Eq. (7)].

\section{CONCLUSIONS}

In conclusion, we have presented a detailed analysis of the Raman spectrum of defective graphene by focusing on the effect of the excitation energy, amount and type of defects. The overall evolution of the Raman spectrum for increasing disorder is similar to that one observed for disordered carbons, although a third stage has not been observed in the case of graphene. By comparing the Raman intensities measured for defective graphene samples containing specific defects, we observed that $I(D) / I(G)$ is not sensitive to the nature of the defects (at least within the spectrometer resolution and for 
the defects analyzed in this work). Thus, the relation between $I(D) / I(G)$ and defect concentration found for vacancies ${ }^{33}$ can be extended to other defects. In contrast, $I\left(D^{\prime}\right) / I(G)$ shows a strong dependence on the type of defect. By using the local activation model, we attribute the different sensitivity of the $D$ and $D^{\prime}$ peaks to the nature of defects to the parameter $C_{S}$ : This is negligible for the $D$ peak, for all the defects considered in this work, in contrast to $C_{S}$ of the $D^{\prime}$ peak. In particular, this is larger for vacancies than $s p^{3}$ sites. This makes $I\left(D^{\prime}\right) / I(G)$ more sensitive to vacancies than $s p^{3}$ sites. As a consequence, the ratios $I(D) / I\left(D^{\prime}\right)$ or $I\left(D^{\prime}\right) / I(G)$ are especially useful to determine the type of defects at a given wavelength. Finally, we have shown that also the energy dependence of $I(D) / I\left(D^{\prime}\right)$ may be affected by the nature of the defects.
In conclusion, this work offers a full insight into the defect-activated Raman scattering process, which will be useful to improve our understanding and modeling of defects in graphene.

\section{ACKNOWLEDGMENTS}

The authors acknowledge useful discussions with A. Jorio, F. Mauri, P. May, and K. S. Novoselov. C.C. acknowledges funding by the Alexander von Humboldt Foundation in the framework of the Sofja Kovalevskaja Award, endowed by the Federal Ministry of Education and Research of Germany. The authors thank S. Reich for giving access to the Dilor spectrometer. *cinzia.casiraghi@manchester.ac.uk

${ }^{1}$ A. K. Geim, Science 324, 1530 (2009).

${ }^{2}$ A. H. Castro Neto, F. Guinea, N. M. R. Peres, K. S. Novoselov, and A. K. Geim, Rev. Mod. Phys. 81, 109 (2009).

${ }^{3}$ K. S. Novoselov, Rev. Mod. Phys. 83, 837 (2011).

${ }^{4}$ A. K. Geim, Rev. Mod. Phys. 83, 851 (2011).

${ }^{5}$ S. V. Morozov, K. S. Novoselov, M. I. Katsnelson, F. Schedin, D. Elias, J. A. Jaszczak, and A. K. Geim, Phys. Rev. Lett. 100, 016602 (2008).

${ }^{6}$ X. Du, I. Skachko, A. Barker, and E. Y. Andrei, Nat. Nanotechnology 3, 491 (2008).

${ }^{7}$ K. I. Bolotin, K. J. Sikes, J. Hone, H. L. Stormer, and P. Kim, Phys. Rev. Lett. 101, 096802 (2008).

${ }^{8}$ A. S. Mayorov, R. V. Gorbachev, S. V. Morozov, L. Britnell, R. Jalil, L. A. Ponomarenko, P. Blake, K. S. Novoselov, K. Watanabe, T. Taniguchi et al., Nano Lett. 11, 2396 (2011).

${ }^{9}$ M. Y. Han, B. Ozyilmaz, Y. Zhang, and P. Kim, Phys. Rev. Lett. 98, 206805 (2007)

${ }^{10}$ Z. Chen, Y.-M. Lin, M. J. Rooks, and P. Avouris, Physica E: Low Dimens. Syst. Nanostruct. 40, 228 (2007).

${ }^{11}$ Y. Zhang, J. P. Small, W. V. Pontius, and P. Kim, Appl. Phys. Lett. 86, 073104 (2005)

${ }^{12}$ M. Lemme, T. Echtermeyer, M. Baus, and H. Kurz, Electron Device Lett. 28, 282 (2007).

${ }^{13}$ Y.-M. Lin, K. A. Jenkins, A. Valdes-Garcia, J. P. Small, D. B. Farmer, and P. Avouris, Nano Lett. 9, 422 (2009).

${ }^{14}$ J. S. Bunch, A. M. van der Zande, S. S. Verbridge, I. W. Frank, D. M. Tanenbaum, J. M. Parpia, H. G. Craighead, and P. L. McEuen, Science 315, 490 (2007).

${ }^{15}$ P. Blake, P. D. Brimicombe, R. R. Nair, T. J. Booth, D. Jiang, F. Schedin, L. A. Ponomarenko, S. V. Morozov, H. F. Gleeson, E. W. Hill et al., Nano Lett. 8, 1704 (2008).

${ }^{16}$ Y. Hernandez, V. Nicolosi, M. Lotya, F. Blighe, Z. Sun, S. D. I. T. McGovern, B. Holland, M. Byrne, Y. Gunko, J. Boland et al., Nat. Nanotechnology 3, 563 (2008).

${ }^{17}$ J. N. Coleman, M. Lotya, A. O’Neill, S. D. Bergin, P. J. King, U. Khan, K. Young, A. Gaucher, S. De, R. J. Smith et al., Science 331, 568 (2011).

${ }^{18}$ G. Eda, G. Fanchini, and M. Chhowalla, Nat. Nanotechnology 3, 270 (2008).

${ }^{19}$ T. Mueller, F. Xia, and P. Avouris, Nat. Photonics 4, 297 (2010).

${ }^{20}$ F. Bonaccorso, Z. Sun, T. Hasan, and A. C. Ferrari, Nat. Photonics 4, 611 (2010).
${ }^{21}$ T. Echtermeyer, L. Britnell, P. Jasnos, A. Lombardo, R. Gorbachev, A. Grigorenko, A. Geim, A. Ferrari, and K. Novoselov, Nat. Commun. 2, 458 (2011).

${ }^{22}$ A. Reina, X. Jia, J. Ho, D. Nezich, H. Son, V. Bulovic, M. S. Dresselhaus, and J. Kong, Nano Lett. 9, 30 (2009).

${ }^{23}$ X. Li, W. Cai, J. An, S. Kim, J. Nah, D. Yang, R. Piner, A Velamakanni, I. Jung, E. Tutuc et al., Science 324, 1312 (2009).

${ }^{24}$ T. Moldt, A. Eckmann, P. Klar, S. V. Morozov, A. A. Zhukov, K. S. Novoselov, and C. Casiraghi, ACS Nano 5, 7700 (2011).

${ }^{25}$ A. Balan, R. Kumar, M. Boukhicha, O. Beyssac, J.-C. Bouillard, D. Taverna, W. Sacks, M. Marangolo, E. Lacaze, R. Gohler et al., Phys. D: Appl. Phys. 43, 374013 (2010).

${ }^{26}$ A. Shukla, R. Kumar, J. Mazher, and A. Balan, Solid State Commun. 149, 718 (2009).

${ }^{27}$ S. Stankovich, D. A. Dikin, R. D. Piner, K. A. Kohlhaas, A. Kleinhammes, Y. Jia, Y. Wu, S. T. Nguyen, and R. S. Ruoff, Carbon 45, 1558 (2007).

${ }^{28}$ J. Zhang, H. Yang, G. Shen, P. Cheng, J. Zhang, and S. Guo, Chem. Commun. 46, 1112 (2010).

${ }^{29}$ H. Yang, Y. Hernandez, A. Schlierf, A. Felten, A. Eckmann, S. Johal, P. Louette, J.-J. Pireaux, X. Feng, K. Mullen et al., Carbon 53, 357 (2013).

${ }^{30}$ C. Berger, Z. Song, X. Li, X. Wu, N. Brown, C. Naud, D. Mayou, T. Li, J. Hass, A. N. Marchenkov et al., Science 312, 1191 (2006).

${ }^{31}$ E. H. Martins Ferreira, M. V. O. Moutinho, F. Stavale, M. M. Lucchese, R. B. Capaz, C. A. Achete, and A. Jorio, Phys. Rev. B 82, 125429 (2010).

${ }^{32}$ L. G. Cancado, A. Jorio, E. H. M. Ferreira, F. Stavale, C. A. Achete, R. B. Capaz, M. V. O. Moutinho, A. Lombardo, T. S. Kulmala, and A. C. Ferrari, Nano Lett. 8, 3190 (2011).

${ }^{33}$ M. Lucchese, F. Stavale, E. M. Ferreira, C. Vilani, M. Moutinho, R. B. C. C. Achete, and A. Jorio, Carbon 48, 1592 (2010).

${ }^{34}$ A. Jorio, E. H. M. Ferreira, M. V. O. Moutinho, F. Stavale, C. A. Achete, and R. B. Capaz, Phys. Status Solidi B 247, 2980 (2010).

${ }^{35}$ H. Wang, Q. Wang, Y. Cheng, K. Li, Y. Yao, Q. Zhang, C. Dong, P. Wang, U. Schwingenschlagl, W. Yang et al., Nano Lett. 12, 141 (2012).

${ }^{36}$ J. J. Lopez, F. Greer, and J. R. Greer, J. Appl. Phys. 107, 104326 (2010).

${ }^{37}$ D. Teweldebrhan and A. A. Balandin, Appl. Phys. Lett. 94, 013101 (2009).

${ }^{38}$ G. Liu, D. Teweldebrhan, and A. Balandin, IEEE Trans. Nanotechnology 10, 865 (2011). 
${ }^{39}$ S. Y. Zhou, C. O. Girit, A. Scholl, C. J. Jozwiak, D. A. Siegel, P. Yu, J. T. Robinson, F. Wang, A. Zettl, and A. Lanzara, Phys. Rev. B 80, 121409 (2009).

${ }^{40}$ D. C. Elias, R. R. Nair, T. M. G. Mohiuddin, S. V. Morozov, P. Blake, M. P. Halsall, A. C. Ferrari, D. W. Boukhvalov, M. I. Katsnelson, A. K. Geim et al., Science 323, 610 (2009).

${ }^{41}$ R. R. Nair, W. Ren, R. Jalil, I. Riaz, V. G. Kravets, L. Britnell, P. Blake, F. Schedin, A. S. Mayorov, S. Yuan et al., Small 6, 2877 (2010).

${ }^{42}$ A. Felten, B. S. Flavel, L. Britnell, A. Eckmann, P. Louette, J.-J. Pireaux, M. Hirtz, R. Krupke, and C. Casiraghi, Small 9, 631 (2013).

${ }^{43}$ D. C. Kim, D.-Y. Jeon, H.-J. Chung, Y. Woo, J. K. Shin, and S. Seo, Nanotechnology 20, 375703 (2009).

${ }^{44}$ M. Jaiswal, C. H. Yi Xuan Lim, Q. Bao, C. T. Toh, K. P. Loh, and B. Ozyilmaz, ACS Nano 5, 888 (2011).

${ }^{45}$ M. Wojtaszek, N. Tombros, A. Caretta, P. H. M. van Loosdrecht, and B. J. van Wees, J. Appl. Phys. 110, 063715 (2011).

${ }^{46}$ I. Childres, L. A. Jauregui, J. Tian, and Y. P. Chen, New J. Phys. 13, 025008 (2011).

${ }^{47}$ Z. Luo, T. Yu, Z. Ni, S. Lim, H. Hu, J. Shang, L. Liu, Z. Shen, and J. Lin, J. Phys. Chem. C 115, 1422 (2011).

${ }^{48}$ W. H. Lee, J. W. Suk, H. Chou, J. Lee, Y. Hao, Y. Wu, R. Piner, D. Akinwande, K. S. Kim, and R. S. Ruoff, Nano Lett. 12, 2374 (2012).

${ }^{49}$ Z. Jin, T. P. McNicholas, C.-J. Shih, Q. H. Wang, G. L. C. Paulus, A. J. Hilmer, S. Shimizu, and M. S. Strano, Chem. Mater. 23, 3362 (2011).

${ }^{50}$ E. Bekyarova, M. E. Itkis, P. Ramesh, C. Berger, M. Sprinkle, W. A. de Heer, and R. C. Haddon, J. Am. Chem. Soc. 131, 1336 (2009).

${ }^{51}$ S. Niyogi, E. Bekyarova, M. E. Itkis, H. Zhang, K. Shepperd, J. Hicks, M. Sprinkle, C. Berger, C. N. Lau, W. A. deHeer et al., Nano Lett. 10, 4061 (2010).

${ }^{52}$ R. Sharma, J. H. Baik, C. J. Perera, and M. S. Strano, Nano Lett. 10, 398 (2010).

${ }^{53}$ J. M. Englert, C. Dotzer, G. Yang, M. Schmid, C. Papp, J. M. Gottfried, H.-P. Steinrck, E. Spiecker, F. Hauke, and A. Hirsch, Nat. Chem. 3, 279 (2011)

${ }^{54}$ Y. A. Kim, K. Fujisawa, H. Muramatsu, T. Hayashi, M. Endo, T. Fujimori, K. Kaneko, M. Terrones, J. Behrends, A. Eckmann et al., ACS Nano 6, 6293 (2012).

${ }^{55}$ L. S. Panchakarla, K. S. Subrahmanyam, S. K. Saha, A. Govindaraj, H. R. Krishnamurthy, U. V. Waghmare, and C. N. R. Rao, Adv. Mater. 21, 4726 (2009).

${ }^{56}$ B. Guo, Q. Liu, E. Chen, H. Zhu, L. Fang, and J. R. Gong, Nano Lett. 10, 4975 (2010).

${ }^{57}$ E. H. Ahlgren, J. Kotakoski, and A. V. Krasheninnikov, Phys. Rev. B 83, 115424 (2011).

${ }^{58}$ J. Lahiri, Y. Lin, P. Bozkurt, I. I. Oleynik, and M. Batzill, Nat. Nanotechnology 5, 326 (2010).

${ }^{59}$ L. D. Carr and M. T. Lusk, Nat. Nanotechnology 316, 5 (2010).

${ }^{60}$ A. C. Ferrari, Solid State Commun. 47143, 47 (2007).

${ }^{61} \mathrm{C}$. Casiraghi, in Spectroscopic Properties of Inorganic and Organometallic Compounds: Techniques, Materials and Applications, edited by J. Yarwood, R. Douthwaite, and S. Duckett (The Royal Society of Chemistry, London, 2012), pp. 29-56.

${ }^{62}$ M. Dresselhaus, A. Jorio, L. G. Cancado, G. Dresselhaus, and R. Saito, in Graphene Nanoelectronics, edited by H. Raza (Springer,
Berlin/Heidelberg, 2012), NanoScience and Technology, pp. $15-55$.

${ }^{63}$ A. Jorio, R. Saito, M. Dresselhaus, and G. Dresselhaus, in Raman Spectroscopy in Graphene Related Systems (Wiley, Weinheim, 2011).

${ }^{64}$ A. C. Ferrari, J. C. Meyer, V. Scardaci, C. Casiraghi, M. Lazzeri, F. Mauri, S. Piscanec, D. Jiang, K. S. Novoselov, S. Roth et al., Phys. Rev. Lett. 97, 187401 (2006).

${ }^{65}$ S. Pisana, M. Lazzeri, C. Casiraghi, K. S. Novoselov, A. K. Geim, A. C. Ferrari, and F. Mauri, Nature Mat. 6, 198 (2007).

${ }^{66}$ A. Das, S. Pisana, S. Piscanec, B. Chakraborty, S. K. Saha, U. V. Waghmare, R. Yang, H. R. Krishnamurhthy, A. K. Geim, A. C. Ferrari et al., Nat. Nanotechnology 3, 210 (2008).

${ }^{67}$ C. Casiraghi, S. Pisana, K. S. Novoselov, A. K. Geim, and A. C. Ferrari, Appl. Phys. Lett. 91, 233108 (2007).

${ }^{68}$ T. M. G. Mohiuddin, A. Lombardo, R. R. Nair, A. Bonetti, G. Savini, R. Jalil, N. Bonini, D. M. Basko, C. Galiotis, N. Marzari et al., Phys. Rev. B 79, 205433 (2009).

${ }^{69}$ J. Zabel, R. R. Nair, A. Ott, T. Georgiou, A. K. Geim, K. S. Novoselov, and C. Casiraghi, Nano Lett. 12, 617 (2012).

${ }^{70}$ C. Casiraghi, Phys. Status Solidi Rapid Res. Lett. 3, 175 (2009).

${ }^{71}$ A. Eckmann, A. Felten, A. Mishchenko, L. Britnell, R. Krupke, K. S. Novoselov, and C. Casiraghi, Nano Lett. 12, 3925 (2012).

${ }^{72}$ C. Casiraghi, A. Hartschuh, H. Qian, S. Piscanec, C. Georgi, A. Fasoli, K. S. Novoselov, D. M. Basko, and A. C. Ferrari, Nano Lett. 9, 1433 (2009).

${ }^{73}$ L. G. Cancado, M. A. Pimenta, B. R. A. Neves, M. S. S. Dantas, and A. Jorio, Phys. Rev. Lett. 93, 247401 (2004).

${ }^{74}$ F. Tuinstra and J. L. Koenig, J. Chem. Phys. 53, 1126 (1970).

${ }^{75}$ F. Tuinstra and J. L. Koenig, J. Comp. Mater. 4, 492 (1970).

${ }^{76}$ R. Nemanich and S. Solin, Solid State Commun. 23, 417 (1977).

${ }^{77}$ R. J. Nemanich and S. A. Solin, Phys. Rev. B 20, 392 (1979).

${ }^{78}$ P. Lespade, A. Marchard, M. Couzi, and F. Cruege, Carbon 22, 375 (1984).

${ }^{79}$ P. Lespade, R. Al-Jishi, and M. Dresselhaus, Carbon 20, 427 (1982).

${ }^{80}$ R. P. Vidano, D. B. Fischbach, L. J. Willis, and T. M. Loehr, Solid State Commun. 39, 341 (1981).

${ }^{81}$ L. G. Canado, K. Takai, T. Enoki, M. Endo, Y. A. Kim, H. Mizusaki, A. Jorio, L. N. Coelho, R. Magalhes-Paniago, and M. A. Pimenta, Appl. Phys. Lett. 88, 163106 (2006).

${ }^{82}$ K. Sato, R. Saito, Y. Oyama, J. Jiang, L. Canado, M. Pimenta, A. Jorio, G. Samsonidze, G. Dresselhaus, and M. Dresselhaus, Chem. Phys. Lett. 427, 117 (2006).

${ }^{83}$ A. C. Ferrari and J. Robertson, Phys. Rev. B 61, 14095 (2000).

${ }^{84}$ A. C. Ferrari and J. Robertson, Phys. Rev. B 64, 075414 (2001).

${ }^{85}$ C. Casiraghi, A. C. Ferrari, and J. Robertson, Phys. Rev. B 72, 085401 (2005).

${ }^{86}$ M. Dresselhaus, G. Dresselhaus, R. Saito, and A. Jorio, Phys. Rep. 409, 47 (2005).

${ }^{87}$ A. Jorio, M. Dresselhaus, and G. Dresselhaus, in Carbon Nanotubes: Advanced Topics in the Synthesis, Structure, Properties and Applications (Springer, Berlin, 2008).

${ }^{88} \mathrm{~S}$. Reich, C. Thomsen, and J. Maultzsch, in Carbon Nanotubes: Basic Concepts and Physical Properties (Wiley-VCH, Berlin, 2004).

${ }^{89}$ C. Thomsen and S. Reich, Phys. Rev. Lett. 85, 5214 (2000). 
${ }^{90}$ D. M. Basko, Phys. Rev. B 78, 125418 (2008).

${ }^{91}$ M. Cardona, in Light Scattering in Solids II (Springer, Berlin, 1982).

${ }^{92}$ P. Venezuela, M. Lazzeri, and F. Mauri, Phys. Rev. B 84, 035433 (2011).

${ }^{93}$ Y. Wang, D. C. Alsme, and R. L. McCreery, Chem. Mater. 2:5, 557 (2009).

${ }^{94}$ I. Pocsik, M. Hundhausen, M. Koos, and L. Ley, J. Non-Cryst. Solids 227, 1083 (1998)

${ }^{95}$ S. Piscanec, M. Lazzeri, F. Mauri, A. C. Ferrari, and J. Robertson, Phys. Rev. Lett. 93, 185503 (2004).

${ }^{96}$ X. Mei, H. Zheng, and J. Ouyang, J. Mater. Chem. 22, 9109 (2012).

${ }^{97}$ D. Yang, A. Velamakanni, G. Bozoklu, S. Park, M. Stoller, R. D. Piner, S. Stankovich, I. Jung, D. A. Field, C. A. Ventrice, Jr. et al., Carbon 47, 145 (2009).
${ }^{98}$ D. L. Mafra, G. Samsonidze, L. M. Malard, D. C. Elias, J. C. Brant, F. Plentz, E. S. Alves, and M. A. Pimenta, Phys. Rev. B 76, 233407 (2007).

${ }^{99}$ D. W. Boukhvalov, M. I. Katsnelson, and A. I. Lichtenstein, Phys. Rev. B 77, 035427 (2008).

${ }^{100}$ A. Nourbakhsh, M. Cantoro, T. Vosch, G. Pourtois, F. Clemente, M. H. van der Veen, J. Hofkens, M. M. Heyns, S. D. Gendt, and B. F. Sels, Nanotechnology 21, 435203 (2010).

${ }^{101}$ M. A. Pimenta, G. Dresselhaus, M. S. Dresselhaus, L. G. Cancado, A. Jorio, and R. Saito, Phys. Chem. Chem. Phys. 9, 1276 (2007).

${ }^{102}$ E. B. Barros, H. Son, G. G. Samsonidze, A. G. Souza Filho, J. Mendes Filho, G. Dresselhaus, and M. S. Dresselhaus, Phys. Rev. B 76, 035444 (2007).

${ }^{103}$ M. Lazzeri, C. Attaccalite, L. Wirtz, and F. Mauri, Phys. Rev. B 78, 081406 (2008). 